\title{
Estimating transiting exoplanet masses from precise optical photometry
}

\author{
D. Mislis ${ }^{1}$, R. Heller ${ }^{2}$, J. H. M. M. Schmitt ${ }^{3}$, and S. Hodgkin ${ }^{1}$ \\ ${ }^{1}$ Institute of Astronomy, Madingley Road, Cambridge CB3 OHA, UK \\ e-mail: misldim@ast.cam.ac.uk \\ 2 Leibniz-Institut für Astrophysik Potsdam (AIP), An der Sternwarte 16, 14482 Potsdam, Germany \\ ${ }^{3}$ Hamburger Sternwarte, Gojenbergsweg 112, 21029 Hamburg, Germany
}

Received 14 February 2011 / Accepted 9 December 2011

\section{ABSTRACT}

\begin{abstract}
We present a theoretical analysis of the optical light curves (LCs) for short-period high-mass transiting extrasolar planet systems. Our method considers the primary transit, the secondary eclipse, and the overall phase shape of the LC between the occultations. Phase variations arise from (i) reflected and thermally emitted light by the planet; (ii) the ellipsoidal shape of the star due to the gravitational pull of the planet; and (iii) the Doppler shift of the stellar light as the star orbits the center of mass of the system. Our full model of the out-of-eclipse variations contains information about the planetary mass, orbital eccentricity, the orientation of periastron and the planet's albedo. For a range of hypothetical systems we demonstrate that the ellipsoidal variations (ii) can be large enough to be distinguished from the remaining components and that this effect can be used to constrain the planet's mass. To detect the ellipsoidal variations, the LC requires a minimum precision of $10^{-4}$, which coincides with the precision of the Kepler mission. As a test of our approach, we consider the Kepler LC of the transiting object HAT-P-7. We are able to estimate the mass of the companion, and confirm its planetary nature solely from the LC data. Future space missions, such as PLATO and the James Webb Space Telescope with even higher photometric precision, will be able to reduce the errors in all parameters. Detailed modeling of any out-of-eclipse variations seen in new systems will be a useful diagnostic tool prior to the requisite ground based radial velocity follow-up.
\end{abstract}

Key words. techniques: photometric - methods: data analysis - planetary systems - methods: analytical - celestial mechanics

\section{Introduction}

Two observational methods have dominated the study of extrasolar planets so far: radial velocity (RV) measurements and transit light curve (LC) analyses. Both have advantages and disadvantages. While RV determinations provide estimates of the planetary mass $\left(M_{\mathrm{p}}\right)$, the eccentricity $(e)$ and the semi-major axis $(a)$, they do not constrain the inclination $(i)$ of the orbital plane with respect to the observer, thus only lower limits to $M_{\mathrm{p}}$ can be determined. The transit method, on the other hand, provides information on $i$, the ratio of the planetary to the stellar radius $\left(R_{\mathrm{p}} / R_{\mathrm{S}}\right)$, and the duration of the transit $(D)$. So far, only a combination of both strategies yields a full set of orbital and physical parameters for extrasolar planets.

Currently, two space-missions are targeted at the detection of transiting extrasolar planets: CoRoT launched in 2006 (Deleuil et al. 1997) and Kepler launched in 2009 (Borucki et al. 1997). Their instruments are monitoring hundreds of thousands of stars, discovering thousands of planet candidates, whose RV followup will take many years (and may never be completed for the faintest candidates). Without RV follow-up, the most fundamental parameter of an extrasolar planet, its mass, remains undetermined from CoRoT and Kepler observations alone. The mass is the crucial parameter classifying an object as a planet, brown dwarf or a star.

High-accuracy photometry has already been used for a number of systems to show that the planetary thermal emission, as well as the reflection of the stellar light from the planet, are detectable. In particular, Welsh et al. (2010) report the discovery of ellipsoidal variations in the Kepler LC of HAT-P-7. This is an effect more commonly known from close stellar systems, where phase-dependent light variation arises from the gravitationally distorted stars. In HAT-P-7, the planet is close enough and massive enough to induce the same effect.

Other authors (Loeb \& Gaudi 2003; Faigler \& Mazeh 2011) have already presented treatments of reflected light, thermal emission, ellipsoidal variations and Doppler beaming. Our approach to the problem includes two main differences: (i) we investigate the sensitivity of our technique on the adopted phase function (Sect. 3.2); and (ii) we attempt to solve separately for the reflected light and thermal emission from the planet. For hot Jupiters around hot stars, the temperature of the planet increases dramatically and reflected light alone fails to explain the LC.

In this paper, we investigate whether the presence of ellipsoidal variations in a planetary system can be used to place meaningful constraints on the mass of the planetary companion. In Sect. 2 we describe the basic phenomena included in our simplified model of a planetary system's LC. In Sect. 3 we compare the magnitudes of the relative contributions and consider the likely range of planetary systems for which ellipsoidal variations ought to be detectable with both CoRoT and Kepler. We reanalyze the HAT-P-7 LC and demonstrate that the ellipsoidal variations can be used to estimate the mass of the planet to within $10 \%$ of the radial-velocity measured mass. We summarize our conclusions in Sect. 4.

\section{Parametrization of photometric transits}

Standard models of LCs that have been used before the advent of space missions are based on a flat curve out of transit and 


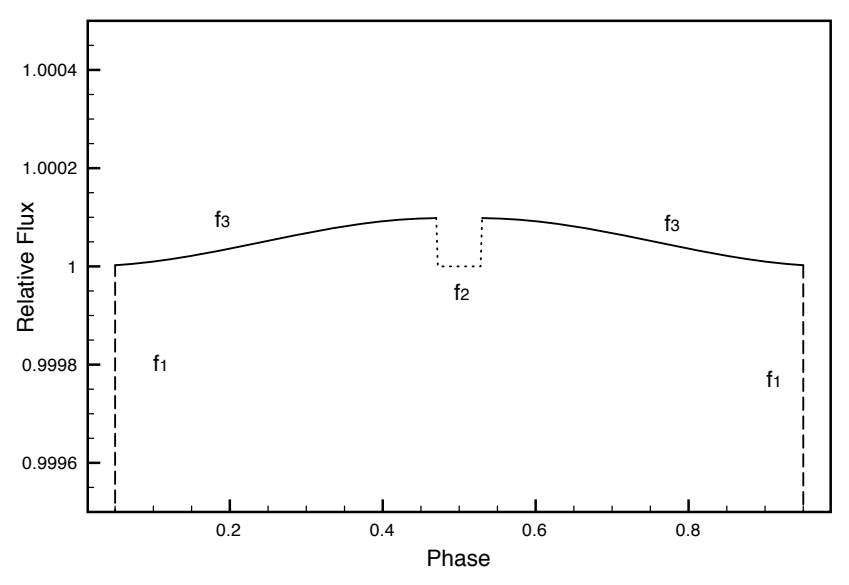

Fig. 1. The standard transit model includes the primary transit $f_{1}$ (dashed line centred at orbital phase $z=0$ ), the secondary eclipse $f_{2}$ (dotted line around $z=0.5$ ) and the reflected light from the planet $f_{3}$ (solid line). The model is based on a HAT-7-b twin planet (Table 2).

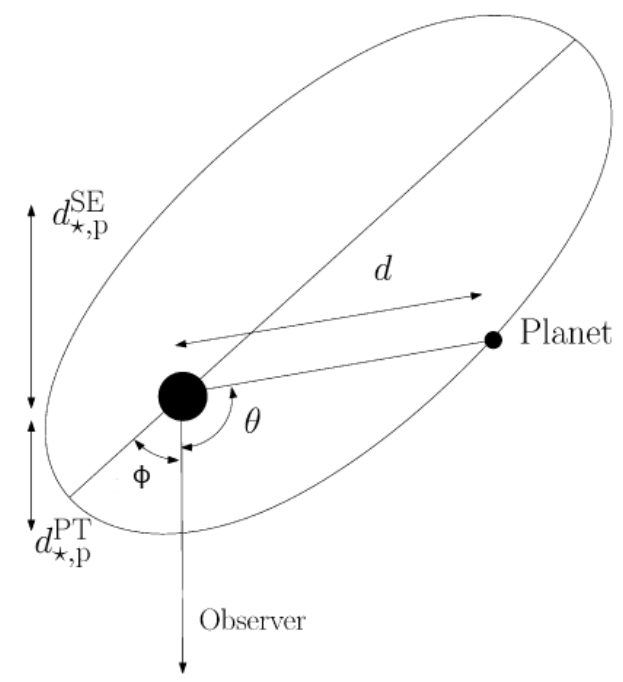

Fig. 2. This sketch of the transiting planetary system, as seen from above, explains the variables used in our calculations. We assume clockwise rotation.

a limb darkening during the transit. Seager \& Mallén-Ornelas (2003) proved analytically that for each package of planet-star characteristics there is a unique LC. Analyses of high-accuracy data from space required a revision of this simple approach. For a high accuracy LC, the model should incorporate the reflected light from the planet, thermal emission, ellipsoidal variations and Doppler boosting, which deforms the overall shape of the LC, and the secondary eclipse (Fig. 1).

In Fig. 2 we show the geometry of an arbitrary transiting system assuming an elliptical orbit. Let $i$ be the angle between the observer's line of sight and the orbital plane normal, while the angle between the observer's line of sight projected onto the orbit plane and the periastron is labeled $\phi$. The star is in the center of the reference frame and $d$ is the distance between the star and the planet; the distance between the star and the planet during the primary transit is denoted by $d_{\star, \mathrm{p}}^{\mathrm{PT}}$, during the secondary eclipse both bodies are separated by the distance $d_{\star, \mathrm{p}}^{\mathrm{SE}}$.

To decode the geometry of the system from the LC we split it into three sub LCs. LC $f_{1}$ describes the course of the primary transit, when the planet blocks the star's light, $f_{2}$ describes the form of the secondary eclipse, when the star blocks and planetary light, and $f_{3}$ the rest of the LC, when both star and planet contribute to the total amount of light.

\subsection{Transit and eclipse}

The duration of the transit $f_{1}$ is given by

$D \simeq \frac{P R_{\star}}{\pi a_{\mathrm{d}}^{2}} \frac{d_{\star, \mathrm{p}}^{\mathrm{PT}}}{\sqrt{1-\mathrm{e}^{2}}} \sqrt{\left(1+\frac{R_{\mathrm{p}}}{R_{\star}}\right)^{2}-b^{2}}$,

where $a_{\mathrm{d}}$ is the semi-major axis of the system, $P$ is the orbital period, $R_{\star}$ and $R_{\mathrm{p}}$ are the radius of the star and the planet, respectively, $e$ is the orbital eccentricity, $b=d_{\star, \mathrm{p}}^{\mathrm{PT}} \cos i / R_{\star}$ is the impact parameter (Ford et al. 2008) and $i$ is the inclination of the orbital plane with respect to the observer's line of sight. To model the shape of $f_{1}$ we use Eq. (1) and the limb darkening equation

$\frac{I_{\mu}}{I_{0}}=1-u_{1}(1-\mu)-u_{2}(1-\mu)^{2}$,

with $u_{1}$ and $u_{2}$ the linear and quadratic limb darkening coefficients respectively (Claret 2004; Sozzetti et al. 2007), $\mu$ as the cosine of the angle between the surface normal and the observer, and $I_{0}$ and $I_{\mu}$ as the intensities at the stellar disk center and at $\mu$, respectively. Once the period is known from observations, one can fit the model to the observations to deduce $R_{\star}, R_{\mathrm{p}}, i$, and $d_{\star, \mathrm{p}}^{\mathrm{PT}}$. The transit of the secondary eclipse, $f_{2}$, is fitted with the same model but without the effects of limb darkening.

\subsection{Phase-dependent light curve}

The shape of the LC between the transit and the eclipse $\left(f_{3}\right)$ contains five contributions: (i) $f_{\star}$, a contribution from the stellar photospheric flux, which almost certainly varies with time, but which we assume to be constant throughout this analysis; (ii) $f_{\text {ref }}$, a contribution from starlight reflected from the face of the planet, which is phase dependent; (iii) $f_{\text {th }}$ contributions from the intrinsic planetary thermal emission (split by day and night), which depends sensitively on a large number of factors, including the spectral range of the observation $\lambda$, the irradiation temperature, and the atmospheric composition and structure; (iv) $f_{\text {ell }}$, a contribution from ellipsoidal variations of the star itself due to tidal forces from the planet; (v) flux variations $f_{\text {dop }}$ due to a Doppler shift (Doppler boosting) of the stellar spectral energy distribution with respect to the bandpass of the instrument (Mazeh \& Faigler 2010). We normalize the LC by the stellar flux, which can be determined as the minimum flux observed at phase $z=0.5$. The phase LC then becomes

$f_{3}(z)=\frac{f_{\star}+f_{\text {ref }}+f_{\text {th, } \mathrm{d}}+f_{\text {th, } \mathrm{n}}+f_{\mathrm{ell}}+f_{\mathrm{dop}}}{f_{\star}}$.

In the following, we study each of these terms in more detail.

\subsubsection{Reflected light}

The phase pattern of the reflected stellar flux depends on the phase angle $z$, i.e., the angle between star and observer as seen from the planet. Counting the orbital phase $\theta$ from primary minimum, the angles $z, i$ and $\theta$ are related through

$\cos (z)=-\sin (i) \cos (\theta)$. 
The reflected flux $f_{\text {ref }}$ can then be expressed as

$f_{\text {ref }}(z)=\alpha_{\mathrm{g}} f_{\star}\left(\frac{R_{\mathrm{P}}}{d}\right)^{2} \Phi(z)$,

where $\alpha_{\mathrm{g}}$ is the geometric albedo of the planet, $f_{\star}$ is the stellar flux at a distance $d$ from the star, and $\Phi(z)$ is the so-called phase function. It is not entirely clear which phase function provides the most appropriate description of extrasolar planets. A popular assumption is

$\Phi_{\mathrm{L}}(z)=\frac{1}{\pi}(\sin z+(\pi-z) \cos z)$

which models the planet as a Lambert sphere (Russell 1916), assuming that the intensity of the reflected light is $\Phi_{\mathrm{L}}=1 / \pi$ at at phase $z=\pi / 2$ (Venus case). An alternative choice is

$\Phi_{\mathrm{C}}(z)=\frac{1}{2}(1+\cos (z))$

which assumes that the reflected light is $\Phi_{\mathrm{C}}=0.5$ at phase $z=$ $\pi / 2$. In our model, we investigate both cases.

The sum $\phi+\theta$ is related to the eccentric anomaly $E$ through

$\phi+\theta=2 \tan ^{-1}\left(\frac{\sqrt{1+e}}{\sqrt{1-e}} \tan (E / 2)\right)$

and $E$ is related to the mean anomaly $M$ through Kepler's equation

$E=M-e \cos (E)$

If $e \neq 0$ then the geometrical albedo is not constant and it is given by the equation Eq. (10) (Kane \& Gelino 2010)

$\alpha_{\mathrm{g}}=\frac{\mathrm{e}^{d-1}-\mathrm{e}^{1-d}}{5\left(\mathrm{e}^{d-1}+\mathrm{e}^{1-d}\right)}+\frac{3}{10}$.

\subsubsection{Ellipsoidal variations}

When a Jupiter-like planet orbits close to its host star, say $a \lesssim 0.1 \mathrm{AU}$, then the star will be distorted. Hence, the skyprojected shape will vary, causing flux variations. Their magnitude is given by

$\frac{f_{\mathrm{ell}}}{f_{\star}}=\beta \frac{M_{\mathrm{p}}}{M_{\star}}\left(\frac{R_{\star}}{a_{\mathrm{d}}}\right)^{3}\left(\frac{1+e \cos (\phi+\theta)}{1-e^{2}}\right)^{3} \sin ^{3}(i)|\sin (\theta)|$,

where

$\beta=\frac{\log _{10}\left(G M_{\star} / R_{\star}^{2}\right)}{\log _{10} T_{\text {eff }}}$

is the gravity darkening term (radiative energy transfer von Zeipel 1924), and $M_{\mathrm{p}}$ and $M_{\star}$ are the masses of the planet and the star, respectively. As these equations show, the detection of the ellipsoidal distortion of the star provides information about the planetary mass with respect to the stellar mass.

\subsubsection{Doppler boosting}

Finally, the relative flux because of stellar Doppler shifts given by

$\frac{f_{\text {dop }}}{f_{\star}}=(3-\rho) \frac{K}{c}$,

with $c$ as the speed of light,

$K=28.4 \cdot P^{-1 / 3} \cdot M_{\mathrm{p}} \cdot \sin (i) \cdot M_{\star}^{-2 / 3}\left(\frac{\sin (\theta)+\mathrm{e} \sin (\phi)}{\sqrt{1-\mathrm{e}^{2}}}\right)$

as the star's velocity amplitude, and $\rho$ given by

$\rho=\frac{e^{h c / k \lambda T_{\text {eff }}\left(3-h c / k \lambda T_{\text {eff }}\right)-3}}{e^{h c / k \lambda T_{\text {eff }}}-1}$.

Explanations of Eqs. (13-15)) and of the underlying phenomenon are given by Loeb \& Gaudi (2003). If this Doppler shift can be measured in addition to the variations induced by the stellar ellipsoidal deformation, then indeed the planetary mass can be constrained independently from the stellar mass.

In the next section, we will firstly explore constraints on accuracy raised by our method and then, as test cases, we will derive the mass of the known transiting exoplanet HAT-P-7, based on Kepler data. Finally, we predict the value of our method for the upcoming ESA mission PLATO.

\subsubsection{Thermal emission}

The equations for the planetary emitted light are similar to those from Cowan \& Agol (2011). Thus

$\frac{f_{\mathrm{th}, \mathrm{d}}(z)}{f_{\star}}=\Phi(z)\left(\frac{R_{\mathrm{p}}}{R_{\star}}\right)^{2} \frac{\mathrm{e}^{h c / \lambda k T_{\text {eff }}}-1}{\mathrm{e}^{h c / \lambda k T_{\mathrm{d}}}-1}$

and

$\frac{f_{\mathrm{th}, \mathrm{n}}(z)}{f_{\star}}=(1-\Phi(z))\left(\frac{R_{\mathrm{p}}}{R_{\star}}\right)^{2} \frac{\mathrm{e}^{h c / \lambda k T_{\text {eff }}}-1}{\mathrm{e}^{h c / \lambda k T_{\mathrm{n}}}-1}$,

$T_{\text {eff }}$ is the effective temperature of the star, and $T_{\mathrm{d}}$ and $T_{\mathrm{n}}$ are the temperatures on the day and night side of the planet, respectively. Using the equations from Hansen (2008) and Cowan \& Agol (2011), we calculate the mean temperature of the planetary day and night sides:

$T_{\mathrm{d}}=T_{\mathrm{o}}\left(1-\alpha_{\mathrm{bol}}\right)^{1 / 4}\left(\frac{2}{3}-\frac{5}{12} \epsilon\right)^{1 / 4}$
$T_{\mathrm{n}}=T_{\mathrm{o}}\left(1-\alpha_{\mathrm{bol}}\right)^{1 / 4}\left(\frac{\epsilon}{4}\right)^{1 / 4}$,

where $T_{\mathrm{o}}=T_{\mathrm{eff}}\left(R_{\star} / d\right)^{0.5}$ and $0 \leq \epsilon \leq 1$ is the energy circulation.

The spectrum of the planet will strongly depend on the absence or presence of clouds, the magnitude of the greenhouse effect and non-grey opacities. For the most of our analysis we assume that the planet emits as a black body. Anyhow, the differences between a black body and a non-black body emission are small (a factor of 1.5-3.5, see Hansen 2008). Assuming main sequence stars and planetary energy circulation $\epsilon=0.0$ (for the maximum thermal emission assuming black bodies), we found that for most hot Jupiters, the thermal emission from the planet is very weak in optical wavelengths. For our analysis we assume a blackbody, observed through the Kepler's sensitivity 

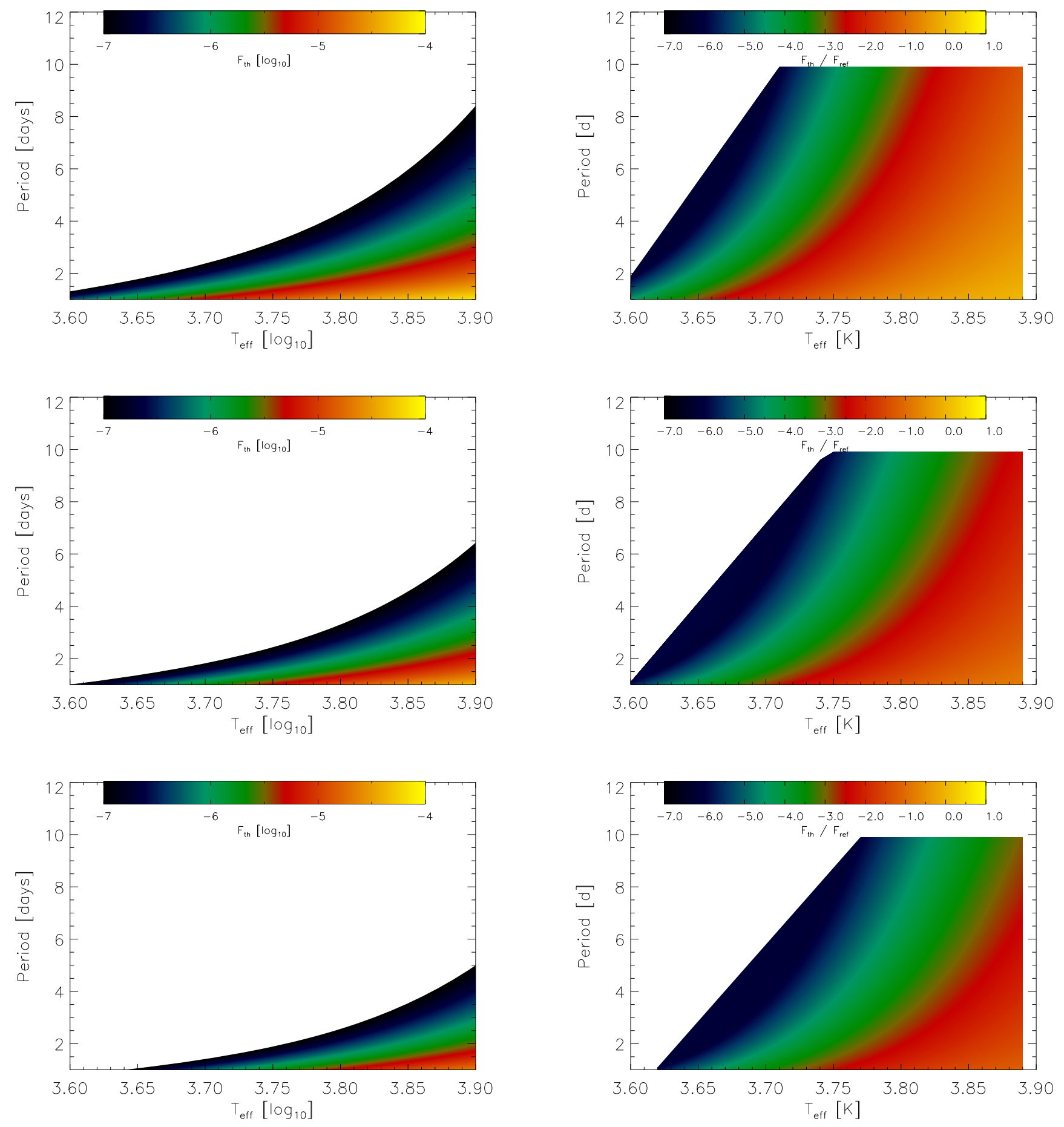

Fig. 3. Thermal emission flux maps for three different bolometric albedo values (top: $\alpha_{\mathrm{bol}}=0.0$, middle: $\alpha_{\mathrm{bol}}=0.3$, bottom: $\alpha_{\mathrm{bol}}=0.5$ ). Thermal emission is stronger for short term period planets around hot stars. For all the plots, we assume $R_{\mathrm{P}}=1.0 R_{\mathrm{J}}$. The white part of the diagram refers to $f_{\text {th }}<10^{-7}$.

curve, which peaks at $575 \mathrm{~nm}$ and covers 420 to $900 \mathrm{~nm}$ (Kepler Handbook). Figure 3 shows the star's effective temperature $T_{\text {eff }}$ versus the period of the planet for three different bolometric albedo $\left(\alpha_{\text {bol }}\right)$ values. Colors refer to the expected stellar relative flux.

The thermal contribution only becomes comparable to, or even larger than, the reflected light component for short period,

low albedo planets around hot stars. Distinguishing the relative contributions from a LC measured in a single passband, without any knowledge of the planet's albedo is hard, if not impossible. Both thermal and reflected components will have similar phase shapes. In Fig. 4 we compare $f_{\text {th }}$ and $f_{\text {ref }}$ for three albedo cases $\left(\alpha_{\mathrm{g}}, \alpha_{\text {bol }}=0.05, \alpha_{\mathrm{g}}, \alpha_{\text {bol }}=0.3, \alpha_{\mathrm{g}}, \alpha_{\text {bol }}=0.5\right)$. 
Table 1. Physical parameters of our two models.

\begin{tabular}{lcc}
\hline \hline Stellar parameter & Hot Jupiter & super-Earth \\
\hline$R_{\star}$ & $1.64 R_{\odot}$ & $0.87 R_{\odot}$ \\
$M_{\star}$ & $1.36 M_{\odot}$ & $0.93 M_{\odot}$ \\
$T_{\text {eff }}$ & $6290 \mathrm{~K}$ & $5275 \mathrm{~K}$ \\
$\beta$ & 0.53 & 0.61 \\
$\epsilon$ & 0.75 & \\
& & \\
Planetary parameter & Hot Jupiter & super-Earth \\
\hline$R_{\mathrm{p}}$ & $1.16 R_{\mathrm{J}}$ & $0.15 R_{\mathrm{J}}$ \\
$M_{\mathrm{p}}$ & $8.75 M_{\mathrm{J}}$ & $0.0151 M_{\mathrm{J}}$ \\
$\alpha_{\mathrm{g}}$ & 0.30 & 0.30 \\
$\alpha_{\text {bol }}$ & 0.65 & 1.0 \\
$u_{1}$ & 0.34 & 0.20 \\
$u_{2}$ & 0.35 & 0.57 \\
& & \\
Orbital parameter & Hot Jupiter & super-Earth \\
\hline$P$ & $5.63347 \mathrm{~d}$ & $0.85360 \mathrm{~d}$ \\
$i$ & $86.72^{\circ}$ & $80.10^{\circ}$ \\
$e$ & 0.52 & 0.05 \\
$\omega$ & $72^{\circ}$ & $5^{\circ}$ \\
\hline
\end{tabular}

Notes. For the super-Earth planet we assume no thermal emission $\left(\alpha_{\text {bol }}=1.0\right)$.

The ellipsoidal variations occur on half the orbital period, and present a rather different phase dependent LC. Even if we cannot accurately distinguish between thermal and reflected components, we show in this paper, that they can be jointly distinguished from ellipsoidal variations (Fig. 7).

\section{Results}

\subsection{Simulations}

To assess the data accuracy required by our method to yield satisfying constraints on the planetary parameters, we simulate two LCs using the equations from above. One of our model planetary systems is a hot Jupiter, analogue to the transiting planet HAT-P-7b (Pál et al. 2009b), whereas the other one resembles the transiting super-Earth CoRoT-7b (Queloz et al. 2009). We customize these models in terms of the geometric albedo, for which we optimistically apply $\alpha_{\mathrm{g}}=0.3$ in both cases (Sudarsky et al. 2000). Though observations of CoRoT-7b are reconcilable with $e=0$, we chose $e=0.05$ to simulate enhanced phase variations in the LC. After all, we are not heading for a reconstruction of these systems but we want to estimate how accurate comparable systems could be parametrized and, as an example, if a putative small eccentricity of CoRoT-7b could be determined. This is particularly important given the significant activity of CoRoT-7b.

To these models, we add increasingly more noise simulating data accuracies between $10^{-7}$ and $10^{-4}$. The phase effect in the LCs, i.e. in $f_{3}$, is significant only for accuracies $\lesssim 10^{-4}$, which is why this phenomenon has not been detected in the LCs of CoRoT (Costes et al. 2004). We then fit the noiseless model from Sect. 2 to each of these - more or less - noisy LCs and use 1000 Monte Carlo simulations to calculate the standard deviations for each parameter in each fit using a bootstrap method explained by Alonso et al. (2008). In Fig. 5 we show the relative errors resulting from these fits for the planetary geometrical albedo $\alpha_{\mathrm{g}}$ $\left(\sigma_{\alpha_{\mathrm{g}}} / \alpha_{\mathrm{g}}\right)$, eccentricity $\left(\sigma_{e} / e\right)$, orientation of periastron $\left(\sigma_{\omega} / \omega\right)$ and the mass ratio $\left(\sigma_{M} / M\right)$ as a function of the root mean square (rms) of the data. With an accuracy provided by Kepler of $10^{-4}$ (Koch et al. 2010), the eccentricity of a CoRoT-7b-like superEarth could merely be determined with a unsatisfactory relative error higher than 1.0 which has no physical use. For a hot Jupiter similar to HAT-P-2b, however, the relative error in $e$ is about 0.001 in the best case (hot Jupiter $-\mathrm{rms} 10^{-7}$ ). The orientation of periastron for the super-Earth could be constrained to approximately $\pm 4^{\circ}$ in the worse case ( $\mathrm{rms} 10^{-4}$ ) while for the hot Jupiter the accuracy is as low as $\pm 0.001^{\circ}$ in the best case $\left(\mathrm{rms} 10^{-7}\right)$. Restrictions of the geometrical albedo $\alpha_{\mathrm{g}}$ are \pm 0.001 in the best case (hot Jupiter $-\mathrm{rms} 10^{-7}$ ) to \pm 1.1 in the worst case (Earth like $-\mathrm{rms} 10^{-4}$ ) with no physical meaning. The respective accuracies in planet mass $M_{\mathrm{P}}$ are $\pm 0.028 M_{\mathrm{J}}$ for hot Jupiters (best case) and $\pm 0.95 M_{\mathrm{J}}$ for super-Earths (worst case).

\subsection{Relative contributions}

Different phase functions (Eqs. (6) and (7)) lead to different peaks in the thermal emission and reflected LC. The ratio between thermal emission and reflected light over ellipsoidal variations becomes smaller (for the Lambert sphere) or larger (for the geometrical sphere), which is why different phase functions lead to different mass values. The mass difference $\left(\Delta M_{\mathrm{P}}\right)$ between the two cases is a constant (36.4\%). For example, if we use a wrong sphere, the mass we measure for HAT-P-7b exoplanet is $\Delta M_{\mathrm{P}} \sim 0.67 M_{\mathrm{J}}$ smaller (geometrical) or larger (Lambert) than the true mass. Seeking out the most plausible phase function, we fit two models, both differing only in the assumption of either the Lambertian or the geometrical sphere, to the data. For most systems, if the components $f_{\text {ref }}, f_{\text {th }}$ and $f_{\text {ell }}$ are very weak, it is impossible to distinguish between the two phase functions. In this case we select the Lambert sphere in order to calculate the upper mass limit of the planet. In Fig. 6 we show the residuals between the two different phase functions. Notice that the shape and the phase ( $x$-axis) of residuals is identical to ellipsoidal variations curve.

For most hot Jupiter systems thermal emission in optical wavelengths is much weaker than variations due to the varying projected shape of the star. For orbital periods of 1 and 5 days of a G0V star, ellipsoidal variations are 3 and 10 times larger than thermal emission, respectively, while $f_{\mathrm{th}} / f_{\star} \sim 10^{-5}$ and $10^{-9}$ (Figs. 3 and 4). As the star becomes hotter (F or A type), or the period shorter ( $<5$ days), the thermal component become to be more significant.

Thermal emission, stellar ellipsoidal deformations, and Doppler shift changes are very weak phenomena (fainter than $10^{-5}$ ), which require very high-accuracy observations to be detected. So far, it has been impossible to detect these effects from the ground but based on data from the recent space-based mission Kepler, our system of equations offers a new gate for exoplanet mass determinations. Neither RV measurements nor transit timing variations are necessary for our procedure.

Our parametrization of reflected and thermal planetary emission are nearly identical, so our model is by definition unable to distinguish between these two effects. Both Eqs. (5) and (16) are only function of the orbital phase $\left(\Phi_{\mathrm{L}, \mathrm{C}}\right)$ and both components (thermal and reflected) provide exactly the same LC. We are not able to distinguish between the two components by fitting our model in a hot Jupiter system, which the planet is tidal locked by its host star. If the planetary rotation and orbital period are different (for a case of a high eccentric orbit and the simple assumption that there are no winds), then reflected light and thermal emission LCs will show different shapes. 

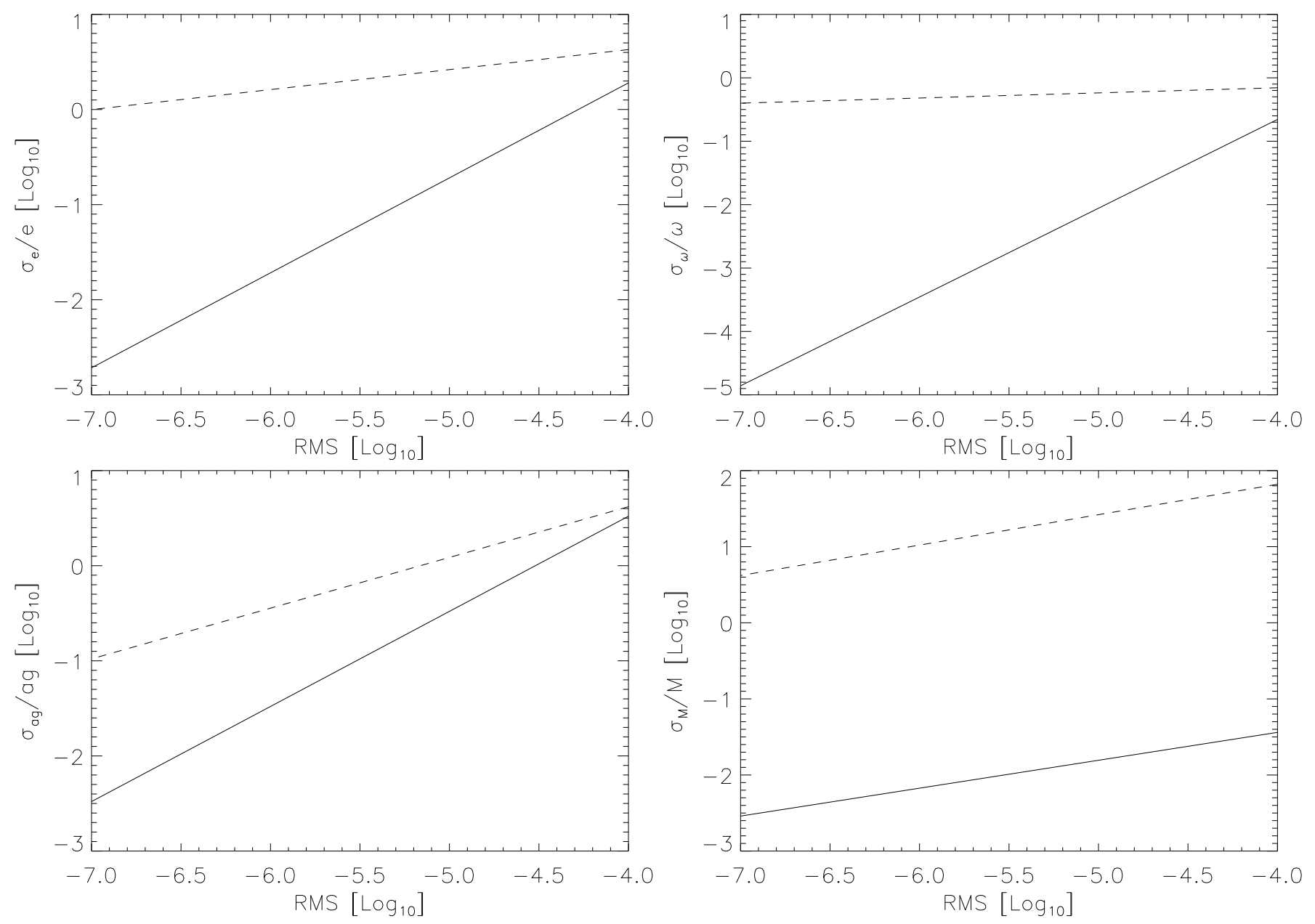

Fig. 5. Relative errors in $e, \omega, \alpha_{\mathrm{g}}$ and $M_{\mathrm{P}}$ as functions of data accuracy. The solid line denotes the HAT-P-2b twin while the dashed line labels the CoRoT-7b analog.

\subsection{Application to Kepler data: the case of HAT-P-7b}

Kepler is a space-based mission, launched on Mar. 7, 2009, with the primary goal to detect Earth-sized and smaller planets in the habitable zone of solar-like stars. For a $m_{V}=12$ star, the photometric precision is $\log _{10}(\mathrm{rms}) \sim-4.35$ for $6 \mathrm{~h}$ combined differential photometry (Borucki et al. 2011). We choose the wellstudied transiting planet HAT-P-7 in the Kepler field, with mass and system parameters constrained by both photometric and RV observations, to test our photometry-only method. We use all the short exposure data (1 min exposures) from Kepler's public archive. Our sample includes 67 transits and the phase folded LC was made by $\sim 180000$ epochs binned by a factor of $\sim 50$.

Welsh et al. (2010) already found ellipsoidal variations in HAT-P-7. Using the established physical parameters of the system, we calculate that the effect of $\left(f_{\text {ell }} \sim 3.1 \times 10^{-5}\right)$ is $\sim 10.3$ times more pronounced than the Doppler shift variations $\left(f_{\text {dop }} \sim 3.0 \times 10^{-6}\right)$. Nevertheless, we include both effects in our model.

We assume that the stellar radius and effective temperature are known $\left(T_{\text {eff }}=6350 \mathrm{~K}\right)$. We fix the ratio of stellar and planetary radius $R_{\mathrm{p}} / R_{\star}=0.0778$, the inclination $i=83.1^{\circ}$ and the relative semi-major axis $a / R_{\star}=8.22$ (Welsh et al. 2010). For our first fit, we use Eq. (3), but we exclude $f_{\text {ell }}$ and $f_{\text {dop. }}$. We only fit $f_{\text {ref }}$ plus $f_{\text {th }}$ in order to check if only these two components could successfully explain the data $\left(\chi_{\text {ref }+ \text { th }}^{2}\right)$. Our second fit includes all four components from Eq. (3) $\left(\chi_{\text {all }}^{2}\right)$. The $\chi^{2}$ ratio between the reflected light plus the thermal emission model and the model which includes all four components is $\chi_{\text {ref }+\mathrm{th}}^{2} / \chi_{\text {all }}^{2}=3.04$. Using an F-test, we found that the complete model explains the data better than the simple model of the reflected plus thermal emission. There is a $0.01 \%$ chance that the more complex model do not produce a better fit to the data (F-Ratio:116.28, $P$-Value: 0.0001). In Fig. 7 we show the observed LC of HAT-P-7 with our best fit as well as a comparison to a model, which includes only reflected stellar light from the planet, and our enhanced model. In Table 2 we list the output parameters of the fit. Because $f_{\text {th }}$ is very weak $\left(10^{-6}-10^{-7}\right.$, see Fig. 3 for the black body case), $\alpha_{\text {bol }}$, $T_{\mathrm{d}}$ and $T_{\mathrm{n}}$ values can not be calculated accurately (very large errors). Welsh et al. (2010), used the secondary eclipse depth of HAT-P-7b, in order to measure the flux from the night side of the planet $\left(f_{\mathrm{th}, \mathrm{n}}=2.2 \times 10^{-5}\right)$. From Fig. 3 (bottom diagram) is clear that the planet is much brighter and much hotter than expected, and other heating mechanisms might exist. We can not disentangle $f_{\text {ref }}$ from $f_{\text {th }}$, however this does not affect our main result.

We find the ratio of the planetary mass over the stellar mass to be $M_{\mathrm{p}} / M_{\star}=1.27 M_{\mathrm{J}} / M_{\odot}$, compared to $M_{\mathrm{p}} / M_{\star}=$ $1.20 M_{\mathrm{J}} / M_{\odot}$ deduced by Welsh et al. (2010). Because the $f_{\text {ell }}$ component is uncorrelated with $f_{\text {ref }}$ and/or $f_{\text {th }}$, the accuracy of the mass ratio $\left(M_{\mathrm{P}} / M_{\star}\right)$ can be calculated with high accuracy. Although our values are not as accurate as those from RV measurements, we can infer the planetary nature of the companion. 
D. Mislis et al.: Transiting exoplanet masses from precise optical photometry

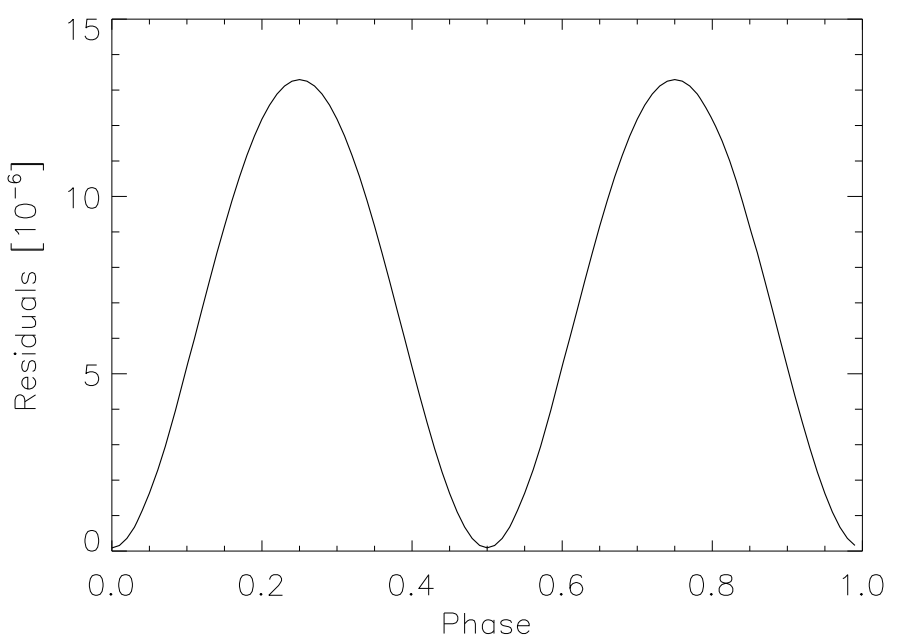

Fig. 6. Residuals flux between Lambert \& geometrical sphere. The residuals curve is identical to the ellipsoidal variations curve. By selecting the wrong phase function we over/under estimate the planetary mass.

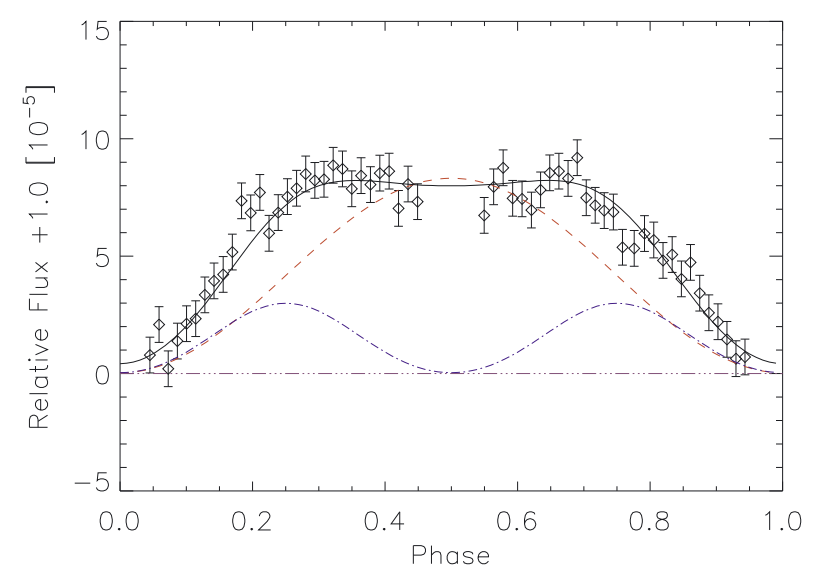

Fig. 7. LC of HAT-P-7. The solid line shows the best fit solution of our synthetic model, i.e. including stellar ellipsoidal distortions and Doppler effect variations. The dashed red line refers to the reflected light component, the blue dotted line refers to the ellipsoidal variations and the purple, almost consant dashed line refers to the thermal emission. The Lambert sphere assumption produced the best fit.

Table 2. HAT-P-7b system parameters.

\begin{tabular}{lcc}
\hline \hline HAT-P-7 parameters & Our model & Other studies \\
\hline$M_{\mathrm{p}} / M_{\star}$ & $1.27 \pm 0.10 M_{\mathrm{J}} / M_{\odot}$ & $1.20 \pm 0.05 M_{\mathrm{J}} / M_{\odot}{ }^{\alpha}$ \\
$e$ & $0.0 \pm 0.1$ & $0.003 \pm 0.012^{\beta}$ \\
$\phi$ & $0.0 \pm 1.0^{o}$ & $0.0^{\beta}$ \\
$\alpha_{\mathrm{g}}$ & $0.21 \pm 0.08$ & $0.18^{\alpha}$ \\
\hline
\end{tabular}

Notes. ${ }^{(\alpha)}$ (Welsh et al. 2010); ${ }^{(\beta)}$ (Pál et al. 2009a).

\subsection{PLATO mission}

PLATO is a prospective ESA mission aiming at the characterization of transiting exoplanets. The mission will use 40 small telescopes covering $42 \times 42 \mathrm{deg}^{2}$ in total with a supposed overall accuracy of $\sim 2.8 \times 10^{-5}$ (Claudi 2010). This will satisfy the needs of our procedure. In the following, we demonstrate the capabilities of our photometry-only method in parameterizing hypothetical exoplanet systems.
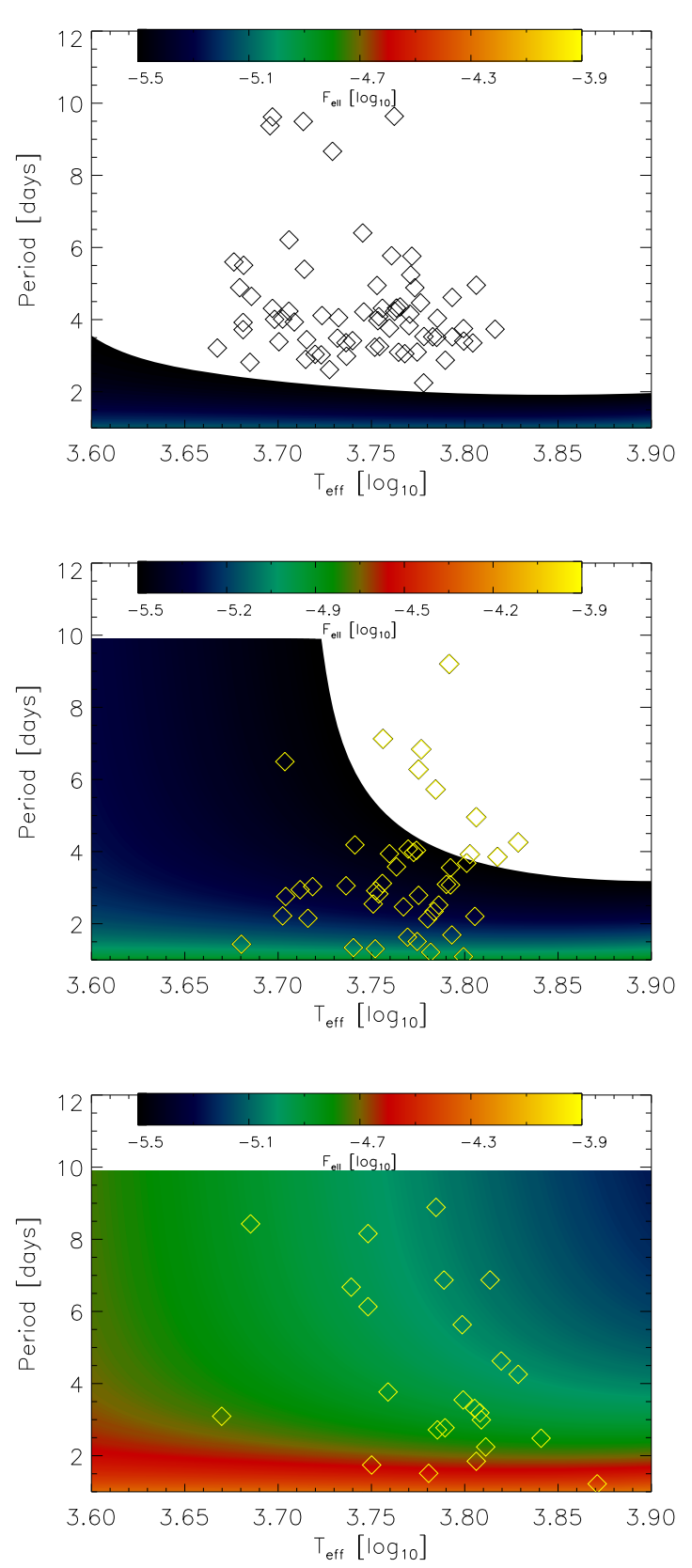

Fig. 8. Combination of stellar ellipsoidal distortion and Doppler shift effects in simulated PLATO observations. The panels show the amplitude of flux variations $\Delta F$ in the LCs for three different planetary masses: $M_{\mathrm{P}}=0.5 M_{\mathrm{J}}$ (top), $1 M_{\mathrm{J}}$ (middle), and $5 M_{\mathrm{J}}$ (bottom) around a range of main-sequence stars. Diamond symbols represent known transiting planets with similar masses. The white part of the diagram refers to $f_{\text {ell }}<10^{-5.5}$.

We investigate the detectability of $f_{\text {ell }}$ and $f_{\text {dop }}$ in a range of different systems, assuming that our target stars belong to the main sequence. For each combination we calculate the amplitude of the flux variations $\Delta F$ in the LC. In Fig. 8 we present three plots, each belonging to a different planetary mass: $M_{\mathrm{p}}=0.5 M_{\mathrm{J}}$ (top), $1 M_{\mathrm{J}}$ (middle), and $5 M_{\mathrm{J}}$ (bottom). For comparison we indicate some known transiting systems with similar masses. In the upper panel $\Delta F$ is dominated by $f_{\text {ell }}$. Here, for the sample of known systems $\Delta F>10^{-5}$, which would be challenging to be detected. Nevertheless, with PLATO one will be able to detect $f_{\text {ell }}$ for planets with $P<3 \mathrm{~d}$ around stars with 
spectral type K0 or later (blue zone in the top panel of Fig. 8). In the middle diagram $\left(M_{\mathrm{p}}=1 M_{\mathrm{J}}\right)$ both $f_{\text {ell }}$ and $f_{\text {dop }}$ affect the LC and $\Delta F \sim 5 \times 10^{-5}$ for most of the known systems - detectable with PLATO. In the bottom panel, where the model planet is most massive, the LCs are mostly affected by the Doppler shift variations rather than by the ellipsoidal geometry of the star. The phenomenon is fairly detectable for the most cases of the known planets while $\Delta F \sim 12 \times 10^{-5}$.

\section{Conclusions}

The mathematical tools presented in this article can be used for a complete parametrization of transiting exoplanet systems on the basis of high-accuracy LCs only. In our model, for a specific range of systems, RV measurements are not necessary to constrain the mass of the planet $\left(M_{\mathrm{p}}\right)$, orbital eccentricity $(e)$, the orientation of periastron $(\omega)$, and the geometric albedo of the planet $\left(\alpha_{\mathrm{g}}\right)$. Our model also incorporates the characterization of the ratio of planetary and stellar radius $\left(R_{\mathrm{p}} / R_{\star}\right)$, orbital period $(P)$, and the orbital inclination $(i)$. In order for this method to be fully applicable, the planet must be massive enough and orbit its host star close enough as to distort the stellar structure significantly.

With the current Kepler and potential future missions such as PLATO, we are able to measure the mass of the hot Jupiters, where the orbital periods must be $\leq 1.5, \leq 4$, and $\leq 10$ days in order to measure planetary masses of $0.5 M_{\mathrm{J}}, 1 M_{\mathrm{J}}$, and $5 M_{\mathrm{J}}$ respectively. Furthermore, for host stars with spectral types earlier than FV, thermal emission component is detectable in the LC (assuming black bodies). This will affect the accuracy and even the detection of ellipsoidal variation.

As we show, the Kepler mission provides an accuracy suitable enough for our procedure to be applied for the characterization of extrasolar planets. We confirm the planetary natures of the hot Jupiters HAT-P-7 from Kepler data alone. Our method yields masses of $M_{\mathrm{p}}=1.27_{-0.45}^{+0.10} M_{\mathrm{J}} \times M_{\star} / M_{\odot}$ for HAT-P-7b. Our results $\left(M_{\mathrm{P}}, \alpha_{\mathrm{g}}, \alpha_{\mathrm{bol}}, e, T_{\mathrm{d}}, T_{\mathrm{n}}\right)$ are in good agreement with Welsh et al. (2010) and we also confirm the ellipsoidal variations of HAT-P-7b system. Using ellipsoidal variations we calculate the planetary mass from the photometric LC itself, without any RV measurments. Our technique will benefit from future space missions such as PLATO and the James Webb Space Telescope (Deming et al. 2009) with rms $\sim 3 \times 10^{-5}$ and $\lesssim 10^{-6}$, respectively.

Acknowledgements. The CoRoT space mission, launched on 2006 December 27, was developed and is operated by the CNES, with participation of the Science Programs of ESA, ESA's RSSD, Austria, Belgium, Brazil, Germany and Spain. This research has made use of NASA's Astrophysics Data System Bibliographic Services.

\section{References}

Alonso, R., Barbieri, M., Rabus, M., et al. 2008, A\&A, 487, L5

Borucki, W. J., Koch, D. G., Dunham, E. W., \& Jenkins, J. M. 1997, in Planets Beyond the Solar System and the Next Generation of Space Missions, ed. D. Soderblom, ASP Conf. Ser., 119, 153

Borucki, W. J., Koch, D. G., Basri, G., et al. 2011, ApJ, 736, 19

Claret, A. 2004, A\&A, 428, 1001

Claudi, R. 2010, Ap\&SS, 328, 319

Costes, V., Bodin, P., Levacher, P., \& Auvergne, M. 2004, in 5th International Conference on Space Optics, ed. B. Warmbein, ESA Spec. Publ., 554, 281

Cowan, N. B., \& Agol, E. 2011, ApJ, 729, 54

Deleuil, M., Barge, P., Leger, A., \& Schneider, J. 1997, in Planets Beyond the Solar System and the Next Generation of Space Missions, ed. D. Soderblom, ASP Conf. Ser., 119, 259

Deming, D., Seager, S., Winn, J., et al. 2009, PASP, 121, 952

Faigler, S., \& Mazeh, T. 2011, MNRAS, 415, 3921

Ford, E. B., Quinn, S. N., \& Veras, D. 2008, ApJ, 678, 1407

Hansen, B. M. S. 2008, ApJS, 179, 484

Kane, S. R., \& Gelino, D. M. 2010, ApJ, 724, 818

Koch, D. G., Borucki, W. J., Basri, G., et al. 2010, ApJ, 713, L79

Loeb, A., \& Gaudi, B. S. 2003, ApJ, 588, L117

Mazeh, T., \& Faigler, S. 2010, A\&A, 521, L59

Pál, A., Bakos, G. Á., Noyes, R. W., \& Torres, G. 2009a, in IAU Symp., 253, 428

Pál, A., Bakos, G. Á., Torres, G., et al. 2009b, MNRAS, 1781

Queloz, D., Bouchy, F., Moutou, C., et al. 2009, A\&A, 506, 303

Russell, H. N. 1916, ApJ, 43, 173

Seager, S., \& Mallén-Ornelas, G. 2003, ApJ, 585, 1038

Sozzetti, A., Torres, G., Charbonneau, D., et al. 2007, ApJ, 664, 1190

Sudarsky, D., Burrows, A., \& Pinto, P. 2000, ApJ, 538, 885

von Zeipel, H. 1924, MNRAS, 84, 665

Welsh, W. F., Orosz, J. A., Seager, S., et al. 2010, ApJ, 713, L145 